\title{
Aufstehen im Sportverein Was Übungsleiter \& Trainer zu einem aktiveren Lebensstil von Kindern beitragen können
}

\author{
H. Hülse ${ }^{1}$
}

\section{Zusammenfassung}

Der Sportverein ist ein Ort, an dem sich Kinder ohnehin sehr viel bewegen. Bezüglich des Weges zum Sport oder der Gewichtung von Theorie und Praxis beim Sport können Überleiter/-innen und Trainer/-innen zusätzlich etwas gegen den Sitzenden Lebensstil unternehmen. Zudem trägt der Sportverein zur Persönlichkeitsentwicklung - und damit zum psychischen und sozialen „Aufstehen“ - bei.

Stichworte: Sitzender Lebensstil, Sportverein, Trainer, Übungsleiter, Selbstbild und Vorbild, Aufstehen

Springen, Hüpfen, Klettern, Rennen Sport ist für viele Kinder eine wunderbare Gelegenheit, sich einmal so richtig auszutoben und nach Herzenslust zu bewegen. Im Sportverein steht „Rumsitzen“ in der Regel nicht auf dem Programm, sondern man steht auf und ist aktiv. Selten kommt es vor, dass man beim Sport dauerhaft sitzt. Sicherlich ist die Zeit, die Kinder im Sportverein verbringen, mit eine der aktivsten. Doch auch im Alltag des Sportvereins gibt es Möglichkeiten für Übungsleiter/-innen oder Trainer/innen, dem insgesamt übermäßig Sitzenden Lebensstil unserer Kinder entgegenzuwirken:

$>$ Wie kommen die Kinder zur Sportstätte?

$>$ Werden sie von den Eltern mit dem Auto gebracht?

$>$ Findet schon auf dem Weg zum Sport die erste Aktivität in Form von Fahrradfahren oder Gehen statt?

> Wie gestalten Übungsleiter/-innen ihre Sportangebote?

${ }^{1}$ Deutsche Sportjugend im Deutschen olympischen Sportbund
$>$ Wie verhält sich der theoretische Anteil zum praktischen, und wie wird dieser umgesetzt?

$>$ Kann ich notwendige Erläuterungen zu Übungsabfolgen statt im Sitzen auch im Stehen durchführen?

> Werden die Erläuterungen statt stationär auch gleich in Bewegung gegeben?

> Gibt es Bewegungsaufgaben, die die Kinder täglich zu Hause in ihren Alltag einbauen können?

Das Setting Sportverein beinhaltet zahlreiche Ansatzpunkte, Kinder rund um den Aufenthalt im Sportverein zu einem aktiveren Lebensstil anzuhalten. Aktivität ist mit körperlichem und seelischem Wohlbefinden verknüpft - der Sportverein kann Kindern ein solch bewegtes Lebensgefühl vermitteln. Vereine sind nicht nur reine Sport- und Bewegungsstätten. Sie sind auch für viele Kinder Treffpunkt mit Freunden. Sportvereine integrieren, inkludieren und prägen - sie verbessern im besten Fall das Selbstbild, ermöglichen Partizipation und machen Kinder stark.

Übungsleiter/-innen haben als Bezugspersonen oft großen Einfluss auf die Kin- der und können sie auch bildlich gesprochen beim „Aufstehen“ unterstützen. Neben der körperlichen Aktivität sollte auch das soziale oder auch emotionale Aufstehen mitgedacht werden. Es geht um ein Signal, die eigenen Belange bewusster in die Hand zu nehmen: Wer sich insgesamt dauerhaft mehr bewegen will, muss eben auch innerlich aufstehen und den Willen zu einem gesünderen und aktiveren Leben entwickeln. Dieses Aufstehen ist umfassender als das rein physische. Es muss sich in allen Lebensbereichen widerspiegeln - angefangen von mehr Alltagsaktivität bis hin zu einer gesunden Ernährungsweise.

Gerade bei Kindern ist es besonders wichtig, dass auch das Umfeld mitspielt. Neben den Eltern, denen eine Schlüsselfunktion zukommt, spielen Bezugspersonen im Verein eine besondere Rolle. Sie können nicht zuletzt als Vorbilder - entscheidende Impulse zu einer veränderten Lebensweise geben. Engagierte im Sport sollten sich dieser besonderen Rolle bewusst sein. Sie können dazu beitragen, Kindern zum ganzheitlich betrachteten Aufstehen und damit zu einem gesünderen und zufriedeneren Leben verhelfen.

Kinder sollen sich wohlfühlen. Wohlbefinden ist unmittelbar mit einem positiven Körpergefühl verknüpft. Nicht nur während der Übungsstunden im Sportverein ist Kinderwelt Bewegungswelt!

Online zu finden unter

http://dx.doi.org/

10-1055/s-0034-1373873 


\section{Summary}

„Getting up“ in a sports club: How physical exercise managers and trainers can contribute to a more active lifestyle for children

The sports club is the place where children are involved in increased physical exercise. Sports managers and trainers are capable of contributing to the reduction of a sedentary lifestyle in children with regard to the process of embracing sport or looking for a balance between theory and practice in sports. The sports club also contributes to the personality development and thus, to psychological and social "getting up “ in children.

Korrespondenzadresse Heike Hülse Deutsche Sportjugend im Deutschen Olympischen Sportbund Ressort Jugendarbeit im Sport

Otto-Fleck-Schneise 12

60528 Frankfurt

Tel. 069/6700308

E-Mail: huelse@dsj.de

Key words: sedentary lifestyle, sport club, trainer, facilitator, self image, role model, standing up 